\title{
A Robust and Effective Method for Solving Two-Point BVP in Modelling Viscoelastic Flows
}

\author{
Mohamed El-Gamel, Ola Mohamed, Neveen El-Shamy \\ Department of Mathematical Sciences, Faculty of Engineering, Mansoura University, Mansoura, Egypt \\ Email: gamel_eg@yahoo.com
}

How to cite this paper: El-Gamel, M. Mohamed, O. and El-Shamy, N. (2020) A Robust and Effective Method for Solving Two-Point BVP in Modelling Viscoelastic Flows. Applied Mathematics, 11, 23-34. https://doi.org/10.4236/am.2020.111003

Received: November 18, 2019

Accepted: January 4, 2020

Published: January 7, 2020

Copyright $\odot 2020$ by author(s) and Scientific Research Publishing Inc. This work is licensed under the Creative Commons Attribution International License (CC BY 4.0).

http://creativecommons.org/licenses/by/4.0/

\begin{abstract}
Chebyshev collocation method is used to approximate solutions of two-point BVP arising in modelling viscoelastic flow. The scheme is tested on four nonlinear problems. The comparison with other methods is made. The results demonstrate the reliability and efficiency of the algorithm developed.
\end{abstract}

\section{Keywords}

Chebyshev, Collocation, Viscoelastic Flow, Fifth-Order, Nonlinear

\section{Introduction}

In modelling viscoelastic flows, differential equations of elliptic-hyperbolic operator types arise. Simulations of such flows have been studied extensively lately. The main characteristics of such elliptic-hyperbolic operators can be captured in a nonlinear fifth order two-point boundary value problem in one dimension [1] [2]. The elasticity parameter in the problem is of major importance in the investigation; that is, if it is increased, then depending on the formulation of the problem.

Many researchers have discussed solutions of viscoelastic flow model, like the Galerkin method discussed in [1], collocation method discussed in [2], the RungKutta discussed in [3], the homotopy method discussed in [4]. Hermitian finite elements in [5] and the shooting method in [6] [7] [8]. Some authors have discussed other methods of solution of this model in [9] [10] [11].

In this paper, we are going to introduce Chebyshev-collocation method for the numerical solution of the fifth-order non-linear two-point boundary value problem in modelling viscoelastic flow:

$$
\left[1+\varepsilon \frac{\mathrm{d} y}{\mathrm{~d} x} \frac{\mathrm{d}}{\mathrm{d} x}\right] \frac{\mathrm{d}^{4} y}{\mathrm{~d} x^{4}}=f(x), \quad-\frac{1}{2} \leq x \leq \frac{1}{2}
$$


concerned to the posterior boundary conditions

$$
\begin{aligned}
& y\left( \pm \frac{1}{2}\right)=\frac{\mathrm{d} y}{\mathrm{~d} x}\left( \pm \frac{1}{2}\right)=0 \\
& \frac{\mathrm{d}^{2} y}{\mathrm{~d} x^{2}}\left(-\frac{1}{2}\right)=c \quad \text { at } \varepsilon>0 .
\end{aligned}
$$

with regard to the given positive constants $\varepsilon$ and $c$ which act as elasticity parameter and a boundary stress, respectively. Moreover, $c$ is equal unity in this paper.

In recent years, a lot of attention has been devoted to the study of Chebyshev methods to investigate various scientific models. Using these methods made it possible to solve Troeschs problem [12], twelfth-order boundary-value problems [13], high-order nonlinear ordinary differential equations [14], linear integrodifferential equations [15], fourth-order Sturm-Liouville problems [16], Generalized Sturm-Liouville problems [17], the parabolic inverse problem [18], twodimensional heat equation [19], fractional diffusion equation [20], elliptic partial differential equations [21], integral and integro-differential equations of the third kind [22], the constant mobility Cahn-Hilliard equation in a square domain [23], Lane-Emden problem [24]. Recently, has been made numerical comparison of sinc-collocation and Chebychev-collocation methods for determining the eigenvalues of Sturm-Liouville problems with parameter-dependent boundary conditions by El-Gamel [25].

Chebyshev methods for ordinary differential equations have many salient features due to the properties of the basis functions and the manner in which the problem is discretized. The approximating discrete system depends only on parameters of the differential equation. There are many advantages of using Chebyshev polynomials as expansion function presented in that are good representations of smooth functions. What we do here is to seek a special Chebyshev solution which also satisfies the given boundary conditions.

We organize our paper as follows. In Section 2, we present the preliminaries which we used in this paper. Method of the solution is given in Section 3. Some numerical results are presented in Section 4 to show the efficiency of the proposed method. Finally, we draw some conclusions and closing remarks.

\section{Essential Relations}

Chebyshev polynomial formula of the first kind of degree $m$ is chiefly defined and bounded in interval $[-1,1]$ see $[26]$

$$
T_{m}(x)=\cos \left(m \cos ^{-1}(x)\right), \quad x=\cos \phi, \quad \phi \in[0, \pi]
$$

or, in didactic organism,

$$
T_{m}(\cos \phi)=\cos (m \phi), \quad m=0,1, \cdots, \quad x \in[-1,1]
$$

As for the shifted Chebyshev polynomial $T^{*}(x)$ of the first kind on interval $[a, b]$.

$$
T_{m}^{*}(x)=T_{m}(q), \quad q=\frac{2}{b-a}\left(x-\frac{a+b}{2}\right) .
$$


With leading coefficient is equal to $2^{m-1}\left(\frac{2}{b-a}\right)^{m}$, by Compensation in the previous equation

$$
T_{m}^{*}(x)=T_{m}\left(\frac{2}{b-a}\left(x-\frac{a+b}{2}\right)\right) .
$$

In addition, the definition of the collocation points is worded as follow

$$
x_{i}=\frac{b-a}{2}\left[\left(\frac{a+b}{b-a}\right)+\cos \left(\frac{i \pi}{N}\right)\right], \quad i=0,1, \cdots, N .
$$

Moreover, the relation between Chebyshev coefficient matrix $\boldsymbol{A}$ and $\boldsymbol{A}^{*(k)}$ in the interval $[a, b]$ is

$$
\boldsymbol{A}^{*(k)}=\left(\frac{4}{b-a}\right)^{k} \boldsymbol{M}^{k} \boldsymbol{A}^{*}
$$

where

$$
\boldsymbol{A}^{*}=\left[\frac{a_{0}^{*}}{2}, a_{1}^{*}, \cdots, a_{N}^{*}\right]^{\tau}
$$

$T=T^{*}$ result from the characteristics of Chebyshev polynomial.

All in all, the use of half interval $-\frac{1}{2} \leq x \leq \frac{1}{2}$ is more favored in modelling viscoelastic flows. The shifted Chebyshev polynomials can also be worded as follows

$$
T_{m}^{*}(x)=T_{m}(2 x)=\cos \left(m \cos ^{-1}(2 x)\right) .
$$

This is deduced from definition of the collocation points:

$$
x_{i}=\frac{1}{2}\left[\cos \left(\frac{i \pi}{N}\right)\right], \quad i=0,1, \cdots, N .
$$

Similarly, the relation between Chebyshev coefficient matrix $\boldsymbol{A}$ and $\boldsymbol{A}^{*(k)}$ in the interval $\left[-\frac{1}{2}, \frac{1}{2}\right]$ is

$$
A^{*(k)}=4^{k} M^{k} A^{*}, \quad k=0,1, \cdots, 5 .
$$

\section{Method of Solution}

Let is assume the approximate solution $y(x)$ of the main problem (1) and its derivatives is

$$
\begin{gathered}
y(x)=\sum_{r=0}^{N} a_{r}^{*} T_{r}^{*}(x), \quad-0.5 \leq x \leq 0.5 \\
y^{(k)}(x)=\sum_{r=0}^{N}\left(a_{r}^{*}\right)^{(k)} T_{r}^{*}(x), \quad k=0,1,2, \cdots, 5 .
\end{gathered}
$$

where $N$ is chosen as any positive integer such that $0 \leq r \leq N$. Besides, the anonymous Chebychev coefficients of $y(x)$ and its derivatives are $a_{r}$ and $a_{r}^{(k)}$, respectively. The approximate solution and its derivatives in the matrix format 
are

$$
\begin{aligned}
& {[y(x)]=\boldsymbol{T}^{*}(x) \boldsymbol{A}^{*}} \\
& {\left[y^{(k)}(x)\right]=\boldsymbol{T}^{*}(x)\left(\boldsymbol{A}^{*}\right)^{k}=4^{k} \boldsymbol{T}^{*}(x) \boldsymbol{M}^{k} \boldsymbol{A}^{*} .}
\end{aligned}
$$

whereas the definitions of ultimate matrices are:

$$
\boldsymbol{Y}=\left[\begin{array}{c}
y\left(x_{0}\right) \\
y\left(x_{1}\right) \\
\vdots \\
y\left(x_{N}\right)
\end{array}\right], \quad \boldsymbol{Y}^{(k)}=\left[\begin{array}{c}
y^{(k)}\left(x_{0}\right) \\
y^{(k)}\left(x_{1}\right) \\
\vdots \\
y^{(k)}\left(x_{N}\right)
\end{array}\right], \quad \boldsymbol{T}=\left[\begin{array}{c}
T\left(x_{0}\right) \\
T\left(x_{1}\right) \\
\vdots \\
T\left(x_{N}\right)
\end{array}\right]
$$

and

$$
\boldsymbol{M}=\left[\begin{array}{ccccccc}
0 & \frac{1}{2} & 0 & \frac{3}{2} & \cdots & 0 & N / 2 \\
0 & 0 & 2 & 0 & \cdots & N-1 & 0 \\
0 & 0 & 0 & 3 & \cdots & 0 & N \\
\vdots & \vdots & \vdots & \vdots & \ddots & \vdots & \vdots \\
0 & 0 & 0 & 0 & 0 & \cdots & N \\
0 & 0 & 0 & 0 & 0 & \cdots & 0
\end{array}\right]_{(N+1) \times(N+1)}
$$

for odd $N$.

$$
\boldsymbol{M}=\left[\begin{array}{ccccccc}
0 & \frac{1}{2} & 0 & \frac{3}{2} & \cdots & \frac{N-1}{2} & 0 \\
0 & 0 & 2 & 0 & \cdots & 0 & N \\
0 & 0 & 0 & 3 & \cdots & N-1 & 0 \\
\vdots & \vdots & \vdots & \vdots & \ddots & \vdots & \vdots \\
0 & 0 & 0 & 0 & \cdots & 0 & N \\
0 & 0 & 0 & 0 & \cdots & 0 & 0
\end{array}\right]_{(N+1) \times(N+1)}
$$

for even $N$. We need the following lemma where $\ell$ and $K$ are both positive integer.

Lemma 1. [13] [14] The following relation holds

$$
\begin{aligned}
{\left[\begin{array}{c}
y^{(k)}\left(x_{0}\right) y^{(\ell)}\left(x_{0}\right) \\
y^{(k)}\left(x_{1}\right) y^{(\ell)}\left(x_{1}\right) \\
\vdots \\
y^{(k)}\left(x_{N}\right) y^{(\ell)}\left(x_{N}\right)
\end{array}\right] } & =\left[\begin{array}{cccc}
y^{(k)}\left(x_{0}\right) & 0 & \cdots & 0 \\
0 & y^{(k)}\left(x_{1}\right) & \cdots & 0 \\
\vdots & \vdots & \ddots & \vdots \\
0 & 0 & \cdots & y^{(k)}\left(x_{N}\right)
\end{array}\right]\left[\begin{array}{c}
y^{(\ell)}\left(x_{0}\right) \\
y^{(\ell)}\left(x_{1}\right) \\
\vdots \\
y^{(\ell)}\left(x_{N}\right)
\end{array}\right] \\
& =\overline{\boldsymbol{Y}}^{(k)} \boldsymbol{Y}^{(\ell)} \\
& =4^{k+\ell}\left(\overline{\boldsymbol{T}^{*}} \overline{\boldsymbol{M}}^{k} \overline{\boldsymbol{A}^{*}}\right) \boldsymbol{T}^{*} \boldsymbol{M}^{\ell} \boldsymbol{A}^{*}
\end{aligned}
$$

where

$$
\overline{\boldsymbol{T}^{*}}=\left[\begin{array}{cccc}
T\left(x_{0}\right) & 0 & \cdots & 0 \\
0 & T\left(x_{1}\right) & \cdots & 0 \\
\vdots & \vdots & \ddots & \vdots \\
0 & 0 & \cdots & T\left(x_{N}\right)
\end{array}\right]
$$




$$
\overline{\boldsymbol{A}^{*}}=\left[\begin{array}{cccc}
\boldsymbol{A}^{*} & 0 & \cdots & 0 \\
0 & \boldsymbol{A}^{*} & \cdots & 0 \\
\vdots & \vdots & \ddots & \vdots \\
0 & 0 & \cdots & \boldsymbol{A}^{*}
\end{array}\right] \text {, and } \overline{\boldsymbol{M}}=\left[\begin{array}{cccc}
\boldsymbol{M} & 0 & \cdots & 0 \\
0 & \boldsymbol{M} & \cdots & 0 \\
\vdots & \vdots & \ddots & \vdots \\
0 & 0 & \cdots & \boldsymbol{M}
\end{array}\right] \text {. }
$$

We need the following the theorem:

Theorem 2. If the considered approximate solution of the problem (1) is (7), so that the discrete Chebyshev system is availed by

$$
W A^{*}=F,
$$

where

$$
\boldsymbol{W}=4^{4} \boldsymbol{T}^{*} \boldsymbol{M}^{4}+\varepsilon 4^{6}\left(\overline{\boldsymbol{T}^{*}} \overline{\boldsymbol{M}} \overline{\boldsymbol{A}^{*}}\right) \boldsymbol{T}^{*} \boldsymbol{M}^{5}
$$

Proof. Replacing each term of (1) with the approximation defined in (7) and (10), and applying the collocation points (4) to it.

The matrices for the boundary conditions subjected to Equation (2) are

$$
\begin{aligned}
& \boldsymbol{T}^{*}\left(\frac{1}{2}\right)=4 \boldsymbol{T}^{*}\left(\frac{1}{2}\right) \boldsymbol{M}=0, \\
& \boldsymbol{T}^{*}\left(-\frac{1}{2}\right)=4 \boldsymbol{T}^{*}\left(-\frac{1}{2}\right) \boldsymbol{M}=0, \\
& 4^{2} \boldsymbol{T}^{*}\left(-\frac{1}{2}\right) \boldsymbol{M}^{2}=c
\end{aligned}
$$

Thus, in the matrix $[W ; F]$ we will replace $5_{\text {th }}$ rows by the Equation (12), we have the augmented matrix $[\tilde{W} ; \tilde{F}]$

$$
\tilde{W} A^{*}=\tilde{\boldsymbol{F}} .
$$

Now we will solve a linear system (13) of $N+1$ equations of the $N+1$ unknown coefficients $a_{r}, r=0,1, \cdots, N$. So as to gain the coefficients of the approximate solution $\boldsymbol{A}^{*}$ by the Q-R method.

\section{Algorithm}

- Input (integer) $N$.

- Input (double) tol.

- Input (array) $A_{\text {old }}=A_{0}$ (Initial approximation, $A_{0}$ with $N+1$ dimension, can be chosen so that the boundary conditions are satisfied).

- $\tilde{W}\left(A_{\text {old }}\right) A_{\text {new }}=\tilde{F}$ is a linear algebraic equation system. Then solve this system to find $A_{\text {new }}$.

- If $\left|A_{\text {old }}-A_{\text {new }}\right|<$ tol then $A_{\text {new }}=A$, break (the program is finished).

- Else then $A_{\text {old }} \leftarrow A_{\text {new }}$.

\section{Examples and Comparison}

In this section we give an illustrative example to authenticate the obtained results on Equation (1). The performance of Chebyshev method is measured by the root mean square errors $E_{\text {chebyshev }}$ which is defined as

$$
\left\|E_{\text {Chebyshev }}\right\|=\sqrt{\sum_{i=0}^{N}\left|y_{\text {Exact }}\left(x_{i}\right)-y_{\text {Chebyshev }}\left(x_{i}\right)\right|^{2}}
$$


Example 1: [1] [2] [3] Consider the following fifth order nonlinear two-point BVP

$$
\left[1+\varepsilon \frac{\mathrm{d} y}{\mathrm{~d} x} \frac{\mathrm{d}}{\mathrm{d} x}\right] \frac{\mathrm{d}^{4} y}{\mathrm{~d} x^{4}}=12, \quad-\frac{1}{2} \leq x \leq \frac{1}{2},
$$

whose exact solution is

$$
y(x)=\frac{1}{2}\left(x^{2}-\frac{1}{4}\right)^{2}
$$

Table 1 exhibits a comparison between the root mean square errors obtained by using the present method, Beam function using Galerkin, Beam function using Collocation, Runge-Kutta methods and Chebyfun for Example 1. This comparison shows the strength of the first scheme. Figure 1 demonstrates Chebyshev approximate solution versus the exact solution.

Example 2: [1] [2] [3] Consider the following fifth order nonlinear two-point BVP

$$
\left[1+\varepsilon \frac{\mathrm{d} y}{\mathrm{~d} x} \frac{\mathrm{d}}{\mathrm{d} x}\right] \frac{\mathrm{d}^{4} y}{\mathrm{~d} x^{4}}=-120 x+600 \varepsilon\left(x^{2}-\frac{1}{4}\right)\left(x^{2}-\frac{1}{20}\right), \quad-\frac{1}{2} \leq x \leq \frac{1}{2},
$$

whose exact solution is

$$
y(x)=-x\left(x^{2}-\frac{1}{4}\right)^{2} .
$$

Table 2 represents a comparison between the root mean square errors with the methods in [1] [2] [3]. Table 3 shows a comparison of between the root mean square errors of Chebyshev and Chebyfun at $\varepsilon=10^{-3}$ and $N=5$ for Example 2. The graph of Chebyshev approximate solution and the exact solution of have been plotted in Figure 2 .

Table 1. Comparison of the root mean square errors at $\varepsilon=10^{-1}$ for Example 1 .

\begin{tabular}{cc}
\hline Method & The root mean square errors \\
\hline $\begin{array}{c}\text { Chebyshev, } N=5 \\
\text { Chebyfun }\end{array}$ & $1.743 \mathrm{E}-18$ \\
Runge-Kutta methods, $N=100 \quad[3]$ & $8.664 \mathrm{E}-18$ \\
Beam function using Galerkin, $N=4 \quad[1]$ & $6.899 \mathrm{E}-03$ \\
Beam function using Collocation, $N=4 \quad[2]$ & $0.975 \mathrm{E}-04$ \\
\hline
\end{tabular}

Table 2. Comparison of the root mean square errors for Example 2, for the present method, Galerkin, collocation methods and Runge-Kutta method.

\begin{tabular}{ccccc}
\hline$\varepsilon$ & $\left\|E_{\text {Cheyshev }}\right\|, N=5$ & $\left\|E_{\text {Runge-Kuta }}\right\|, N=100$ & $\left\|E_{\text {Galerkin }}\right\|, N=5$ & $\left\|E_{\text {Collocation }}\right\|, N=5$ \\
& {$[3]$} & {$[1]$} & $0.25 \mathrm{E}-03$ & $0.24 \mathrm{E}-02$ \\
\hline $10^{-1}$ & $4.7300 \mathrm{E}-13$ & $3.98537 \mathrm{E}-03$ & $0.16 \mathrm{E}-03$ & $0.22 \mathrm{E}-02$ \\
$10^{-2}$ & $1.7820 \mathrm{E}-13$ & $3.98585 \mathrm{E}-03$ & $0.16 \mathrm{E}-03$ & $0.22 \mathrm{E}-02$ \\
$10^{-3}$ & $1.2704 \mathrm{E}-16$ & $3.98599 \mathrm{E}-03$ & $-\cdots$ & $-\cdots--$ \\
$10^{-4}$ & $2.0230 \mathrm{E}-16$ & $3.98586 \mathrm{E}-03$ & & \\
\hline
\end{tabular}


Table 3. Comparison of the root mean square errors at $\varepsilon=10^{-3}$ and $N=5$ for Example 2 .

\begin{tabular}{cc}
\hline Method & The root mean square errors \\
\hline Chebyshev & $1.2704 \mathrm{E}-16$ \\
Chebyfun & $2.7800 \mathrm{E}-16$ \\
\hline
\end{tabular}

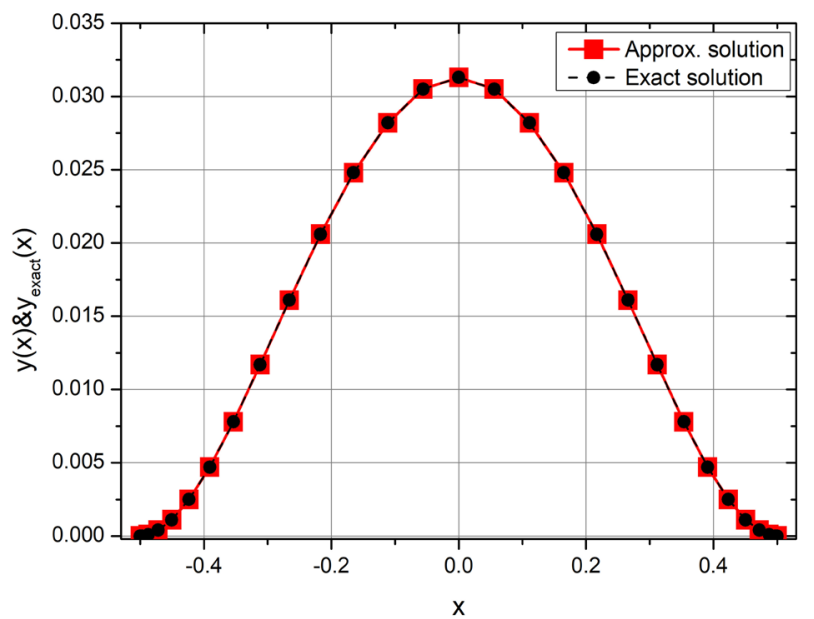

Figure 1. The exact solution and approximation solution for Example 1.

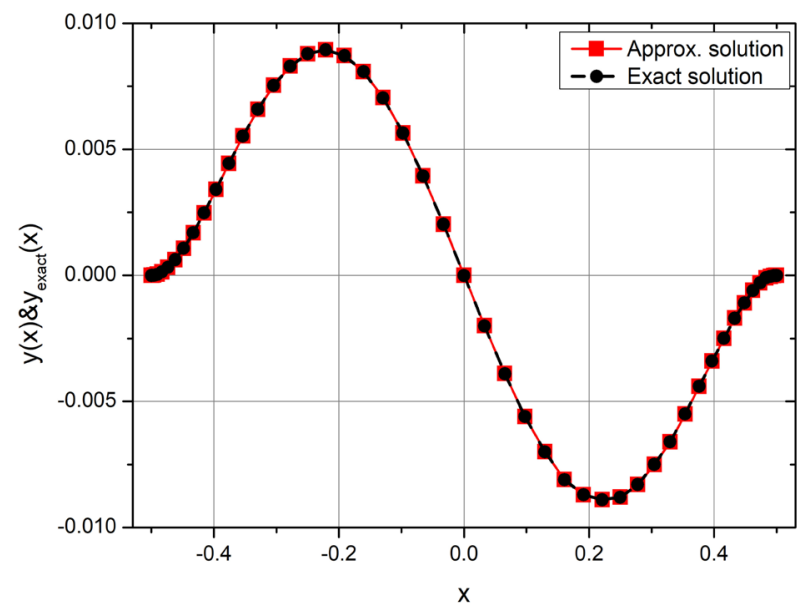

Figure 2. The exact solution and approximation solution for Example 2.

Example 3: [1] [2] [3] Consider the following fifth order nonlinear two-point BVP

$$
\left[1+\varepsilon \frac{\mathrm{d} y}{\mathrm{~d} x} \frac{\mathrm{d}}{\mathrm{d} x}\right] \frac{\mathrm{d}^{4} y}{\mathrm{~d} x^{4}}=f(x), \quad-\frac{1}{2} \leq x \leq \frac{1}{2},
$$

whose exact solution is

$$
y(x)=\frac{1}{2 \pi}\left(x^{2}-\frac{1}{4}\right) \sin \pi\left(x-\frac{1}{2}\right) .
$$

and 


$$
\begin{aligned}
f(x)= & \frac{\pi}{2}\left[\pi^{2}\left(x^{2}-\frac{1}{4}\right)-12\right] \sin \pi\left(x-\frac{1}{2}\right)-4 \pi^{2} x \cos \pi\left(x-\frac{1}{2}\right) \\
& +\frac{1}{8} \varepsilon \pi^{2}\left[\pi^{2}\left(x^{2}-\frac{1}{4}\right)^{2}-40\left(x^{2}-\frac{1}{8}\right)\right] \cos 2 \pi\left(x-\frac{1}{2}\right) \\
& +\frac{1}{8} \varepsilon \pi x\left[12 \pi^{2}\left(x^{2}-\frac{1}{4}\right)-40\right] \sin 2 \pi\left(x-\frac{1}{2}\right) \\
& +\frac{1}{8} \varepsilon \pi^{2}\left[\pi^{2}\left(x^{2}-\frac{1}{4}\right)^{2}+5\right]
\end{aligned}
$$

Tables 4-7 illustrate the comparison between result of Chebyshev polynomial method and result of methods in [1] and [2] at $\varepsilon=10^{-1}, 10^{-3}, 10$ and $10^{3}$ with $N$ from 6 to 16 . Besides, Table 8 depicts $\varepsilon$ with values $10^{-1}, 10^{-2}$ and $10^{-3}$ that has an impact on the result of Chebyshev polynomial method at $N=18$, Chebfun matlab program and the Runge-Kutta in [3] at $N=100$. Figure 3 demonstrates Chebyshev approximate solution versus the exact solution.

Table 4. Comparison of the root mean square errors at $\varepsilon=10^{-1}$ for Example 3.

\begin{tabular}{cccc}
\hline$N$ & $\left\|E_{\text {Chebshhev }}\right\|$ & $\left\|E_{\text {Collocation }}\right\|[2]$ & $\left\|E_{\text {Galerkin }}\right\|[1]$ \\
\hline 6 & $4.3706 \mathrm{E}-04$ & $0.22 \mathrm{E}-02$ & --- \\
8 & $1.0349 \mathrm{E}-05$ & $0.43 \mathrm{E}-04$ & $0.84 \mathrm{E}-02$ \\
10 & $1.1865 \mathrm{E}-07$ & $0.44 \mathrm{E}-06$ & $0.19 \mathrm{E}-06$ \\
14 & $3.8885 \mathrm{E}-12$ & $0.12 \mathrm{E}-10$ & $0.20 \mathrm{E}-09$ \\
16 & $1.2817 \mathrm{E}-14$ & $0.38 \mathrm{E}-13$ & $0.18 \mathrm{E}-09$ \\
\hline
\end{tabular}

Table 5. Comparison of the root mean square errors at $\varepsilon=10^{-3}$ for Example 3 .

\begin{tabular}{cccc}
\hline$N$ & $\left\|E_{\text {Chebyshev }}\right\|$ & $\left\|E_{\text {Collocation }}\right\|[2]$ & $\left\|E_{\text {Galerkin }}\right\|[1]$ \\
\hline 6 & $4.7455 \mathrm{E}-04$ & $0.24 \mathrm{E}-02$ & $0.60 \mathrm{E}-02$ \\
8 & $8.7264 \mathrm{E}-06$ & $0.37 \mathrm{E}-04$ & $0.84 \mathrm{E}-04$ \\
10 & $8.7156 \mathrm{E}-08$ & $0.32 \mathrm{E}-06$ & $0.29 \mathrm{E}-06$ \\
14 & $2.3566 \mathrm{E}-12$ & $0.73 \mathrm{E}-11$ & $0.31 \mathrm{E}-09$ \\
16 & $8.2138 \mathrm{E}-15$ & $0.21 \mathrm{E}-13$ & $0.29 \mathrm{E}-09$ \\
\hline
\end{tabular}

Table 6. Comparison of the root mean square errors at $\varepsilon=10$ for Example 3 .

\begin{tabular}{cccc}
\hline$N$ & $\left\|E_{\text {Chebsshev }}\right\|$ & $\left\|E_{\text {Collocation }}\right\|[2]$ & $\left\|E_{\text {Galerkin }}\right\|[1]$ \\
\hline 6 & $0.19 \mathrm{E}-02$ & $0.95 \mathrm{E}-02$ & --- \\
8 & $1.1786 \mathrm{E}-04$ & $0.11 \mathrm{E}-02$ & $0.27 \mathrm{E}-04$ \\
10 & $2.3777 \mathrm{E}-06$ & $0.91 \mathrm{E}-05$ & $0.16 \mathrm{E}-06$ \\
14 & $1.9856 \mathrm{E}-10$ & $0.69 \mathrm{E}-09$ & $0.13 \mathrm{E}-10$ \\
16 & $1.4004 \mathrm{E}-12$ & $0.41 \mathrm{E}-11$ & $0.13 \mathrm{E}-10$ \\
\hline
\end{tabular}


Table 7. Comparison of the root mean square errors at $\varepsilon=10^{3}$ for Example 3.

\begin{tabular}{cccc}
\hline$N$ & $\left\|E_{\text {Chebyshev }}\right\|$ & $\left\|E_{\text {Collocation }}\right\|[2]$ & $\left\|E_{\text {Galerkin }}\right\|[1]$ \\
\hline 6 & $7.9662 \mathrm{E}-04$ & $0.40 \mathrm{E}-02$ & --- \\
8 & $1.5049 \mathrm{E}-05$ & $0.20 \mathrm{E}-03$ & $0.14 \mathrm{E}-03$ \\
10 & $1.8040 \mathrm{E}-06$ & $0.69 \mathrm{E}-05$ & $0.16 \mathrm{E}-06$ \\
14 & $1.1496 \mathrm{E}-10$ & $0.47 \mathrm{E}-09$ & $0.13 \mathrm{E}-10$ \\
16 & $4.9300 \mathrm{E}-13$ & $0.20 \mathrm{E}-10$ & $0.13 \mathrm{E}-10$ \\
\hline
\end{tabular}

Table 8. Comparison of the root mean square errors for Chebyshev polynomial method and the Runge-Kutta method for Example 3.

\begin{tabular}{cccc}
\hline$\varepsilon$ & $\left\|E_{\text {Chebyshev }}\right\|, N=18$ & $\left\|E_{\text {Chebyfun }}\right\|$ & $\left\|E_{\text {Runge-Kutta }}\right\|, N=100 \quad[3]$ \\
\hline $10^{-1}$ & $1.9836 \mathrm{E}-16$ & $2.84 \mathrm{E}-17$ & $2.52017 \mathrm{E}-02$ \\
$10^{-2}$ & $4.1631 \mathrm{E}-16$ & $1.01 \mathrm{E}-16$ & $9.238658 \mathrm{E}-03$ \\
$10^{-3}$ & $1.0646 \mathrm{E}-15$ & $6.71 \mathrm{E}-16$ & $8.836376 \mathrm{E}-03$ \\
\hline
\end{tabular}

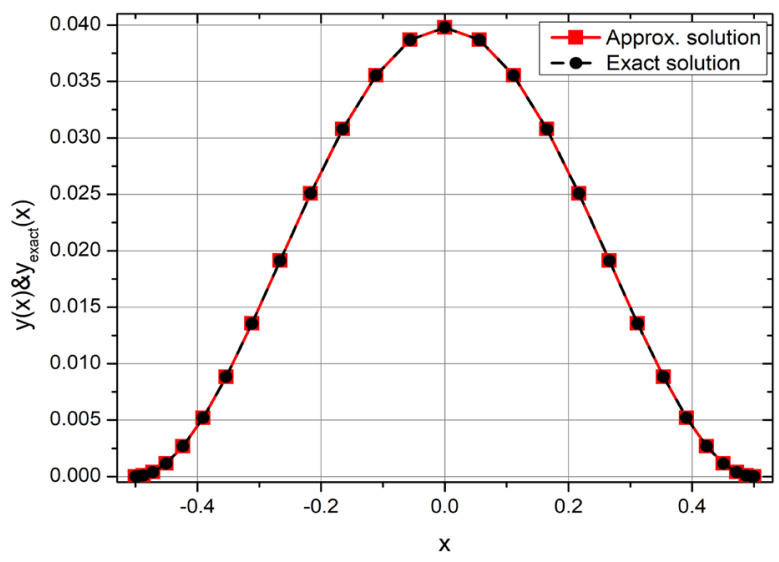

Figure 3. The exact solution and approximation solution for Example 3.

Example 4: Now we turn to a singular problem

$$
\left[1+\varepsilon \frac{\mathrm{d} y}{\mathrm{~d} x} \frac{\mathrm{d}}{\mathrm{d} x}\right] \frac{\mathrm{d}^{4} y}{\mathrm{~d} x^{4}}+\frac{1}{x} y^{\prime}+\frac{1}{x^{2}} y=f(x), \quad-\frac{1}{2} \leq x \leq \frac{1}{2},
$$

and

$$
\begin{aligned}
f(x)= & \varepsilon 1440 x\left[4 x\left(x^{2}-\frac{1}{4}\right)^{2}+8 x^{3}\left(x^{2}-\frac{1}{4}\right)\right]+6\left(x^{2}-\frac{1}{4}\right)^{2} \\
& +8 x^{2}\left(x^{2}-\frac{1}{4}\right)+720 x^{2}-24
\end{aligned}
$$

whose exact solution is

$$
y(x)=2 x^{2}\left(x^{2}-\frac{1}{4}\right)^{2} .
$$

Applying L'Hospital rule to BVP in order to remove singularity. The latter form is 


$$
\begin{gathered}
{\left[1+\varepsilon \frac{\mathrm{d} y}{\mathrm{~d} x} \frac{\mathrm{d}}{\mathrm{d} x}\right] \frac{\mathrm{d}^{4} y}{\mathrm{~d} x^{4}}+p(x) y^{\prime \prime}+d(x) y^{\prime}+b(x) y=f(x)} \\
p(x)=\left\{\begin{array}{ll}
0, & x \neq 0 ; \\
1.5, & x=0 .
\end{array} d(x)=\left\{\begin{array}{ll}
1 / x, & x \neq 0 ; \\
0, & x=0 .
\end{array} \quad b(x)= \begin{cases}1 / x^{2}, & x \neq 0 ; \\
0, & x=0 .\end{cases} \right.\right.
\end{gathered}
$$

The computational results are summarized in Table 9.

Table 10 exhibits a comparison between the root mean square errors at $\varepsilon=10^{-1}$ and $N=6$ for Example 4 obtained by using Chebyshev method and Chebfun. Figure 4 demonstrates Chebyshev approximate solution and the exact which appears to be in good agreement with each other.

\section{Conclusion}

In this article, we present a method to approximate the solution of viscoelastic flows. The numerical method is based on the operational matrix of Chebychev polynomials. We present four examples, the first three examples are nonlinear fifth-order differential equation and fourth example is singular nonlinear. We compared the results of this algorithm with others and showed the accuracy and potential applicability of the given method. The proposed method is a powerful tool for obtaining novel numerical solutions of such equations. It is advisable to use it for other nonlinear differential equations.

Table 9. Comparison of the root mean square errors at $N=6$ for Example 4.

\begin{tabular}{cc}
\hline$\varepsilon$ & $\left\|E_{\text {Chebyshev }}\right\|$ \\
\hline $10^{-1}$ & $8.7184 \mathrm{E}-12$ \\
$10^{-2}$ & $2.9893 \mathrm{E}-14$ \\
$10^{-3}$ & $9.3948 \mathrm{E}-15$ \\
\hline
\end{tabular}

Table 10. Comparison of the root mean square errors at $\varepsilon=10^{-1}$ and $N=6$ for Example 4 .

\begin{tabular}{cc}
\hline Method & The root mean square errors \\
\hline Chebyshev & $8.718 \mathrm{E}-12$ \\
Chebfun & $11.40 \mathrm{E}-11$ \\
\hline
\end{tabular}

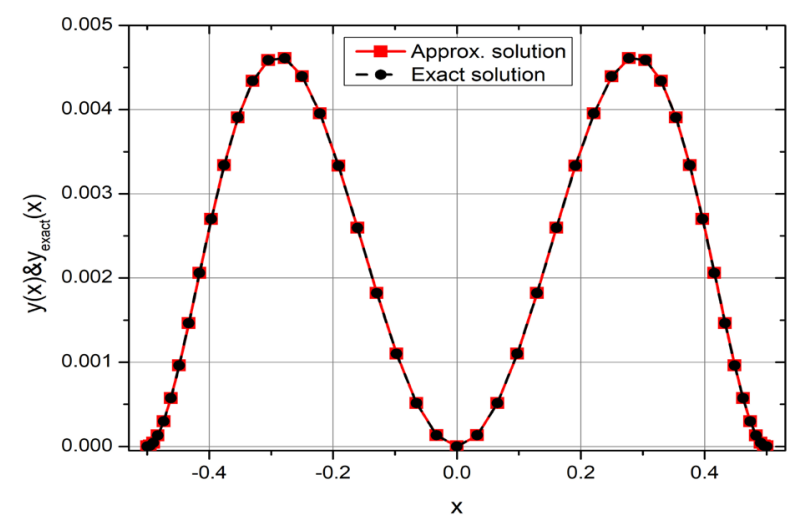

Figure 4. The exact solution and approximation solution for Example 4. 


\section{Conflicts of Interest}

The authors declare no conflicts of interest regarding the publication of this paper.

\section{References}

[1] Davies, A., Karageorghis, A. and Phillips, T. (1988) Spectral Galerkin Methods for the Primary Two-Point Boundary Value Problem in Modelling Viscoelastic Flows. International Journal for Numerical Methods in Engineering, 26, 647-662. https://doi.org/10.1002/nme.1620260309

[2] Davies, A., Karageorghis, A. and Phillips, T. (1988) Spectral Collocation Methods for the Primary Two-Point Boundary Value Problem in Modelling Viscoelastic Flows. International Journal for Numerical Methods in Engineering, 26, 805-813. https://doi.org/10.1002/nme.1620260404

[3] Attili, B. (2000) Initial Value Methods for the Primary Two Point Boundary Value Problems in Modelling Viscoelastic Flows. International Journal of Computer Mathematics, 74, 379-390. https://doi.org/10.1080/00207160008804949

[4] Syam, M. and Attili, B. (2005) Numerical Solution of Singularly Perturbed Fifth Order Two Point Boundary Value Problem. Applied Mathematics and Computation, 170, 1085-1094. https://doi.org/10.1016/j.amc.2005.01.003

[5] Marchal, J. and Crochet, M. (1986) Hermitian Finite Elements for Calculating Viscoelastic Flow. Journal of Non-Newtonian Fluid Mechanics, 20, 187-208. https://doi.org/10.1016/0377-0257(86)80021-0

[6] Attili, S., Elgindi, M. and El-Gebeily, A. (1997) Initial Value Methods for the Eigenelements of Singular Two Point Boundary Value Problems. AJSE, 22, 67-77.

[7] Attili, S. (1993) On the Numerical Implementation of the Shooting Methods to the One Dimensional Singular Boundary Value Problems. International Journal of Computer Mathematics, 47, 65-75. https://doi.org/10.1080/00207169308804163

[8] Elgindi, B. and Langer, W. (1994) On the Shooting Method for a Class of Two Point Singular Nonlinear Boundary Value Problems. International Journal of Computer Mathematics, 51, 107-118. https://doi.org/10.1080/00207169408804270

[9] O’Mally, R. (1974) Introduction to Singular Perturbation. Academic Press, New York.

[10] Kevorkian, J. and Cole, J. (1981) Perturbation Methods in Applied Mathematics. Springer, Berlin. https://doi.org/10.1007/978-1-4757-4213-8

[11] Phillips, T. (1989) On the Potential of Spectral Methods to Solve Problems in NonNewtonian Fluid Mechanics. Numerical Methods for Partial Differential Equations, 5, 35-43. https://doi.org/10.1002/num.1690050104

[12] El-Gamel, M. and Sameeh, M. (2013) A Chebychev Collocation Method for Solving Troesch's Problem. International Journal of Mathematics and Computer Applications Research, 3, 23-32.

[13] El-Gamel, M. (2015) Chebychev Polynomial Solutions of Twelfth-Order BoundaryValue Problems. British Journal of Mathematics \& Computer Science, 6, 13-23. https://doi.org/10.9734/BJMCS/2015/8874

[14] Akyuz-Dascioglu, A. and Erdik-Yaslan, H. (2011) The Solution of High-Order Nonlinear Ordinary Differential Equations by Chebyshev Series. Applied Mathematics and Computation, 217, 5658-5666. https://doi.org/10.1016/j.amc.2010.12.044

[15] Akyz, A. and Sezer, M. (1999) A Chebyshev Collocation Method for the Solution of Linear Integro-Differential Equations. International Journal of Computer Mathemat- 
ics, 72, 491-507. https://doi.org/10.1080/00207169908804871

[16] El-Gamel, M. and Sameeh, M. (2012) An Efficient Technique for Finding the Eigenvalues of Fourth-Order Sturm-Liouville Problems. Applied Mathematics, 3, 920-925. https://doi.org/10.4236/am.2012.38137

[17] El-Gamel, M. and Sameeh, M. (2014) Generalized Sturm-Liouville Problems and Chebychev Collocation Method. British Journal of Mathematics \& Computer Science, 4, 1124-1133. https://doi.org/10.9734/BJMCS/2014/7670

[18] Ranjbar, M. and Aghazadeh, M. (2018) Collocation Method Based on Shifted Chebyshev and Radial Basis Functions with Symmetric Variable Shape Parameter for Solving the Parabolic Inverse Problem. Inverse Problems in Science and Engineering, 27, 369-387. https://doi.org/10.1080/17415977.2018.1462355

[19] Gumgum, S., Kurul, E. and Savasaneril, N. (2018) Chebyshev Collocation Method for the Two-Dimensional Heat Equation. CMMA, 3, 1-8.

[20] Agarwal, P. and El-Sayed, A. (2018) Non-Standard Finite Difference and Chebyshev Collocation Methods for Solving Fractional Diffusion Equation. Physica A, 500, 40-49. https://doi.org/10.1016/j.physa.2018.02.014

[21] Ghimire, B., Lamichhane, A., et al. (2020) Hybrid Chebyshev Polynomial Scheme for Solving Elliptic Partial Differential Equations. Journal of Computational and Applied Mathematics, 364, 1123. https://doi.org/10.1016/j.cam.2019.06.040

[22] Sakran, M. (2019) Numerical Solutions of Integral and Integro-Differential Equations Using Chebyshev Polynomials of the Third Kind. Applied Mathematics and Computation, 351, 66-82. https://doi.org/10.1016/j.amc.2019.01.030

[23] Lee, K. (2020) Chebyshev Collocation Method for the Constant Mobility Cahn-Hilliard Equation in a Square Domain. Applied Mathematics and Computation, 370, Article ID: 124931. https://doi.org/10.1016/j.amc.2019.124931

[24] Sharma, B., Kumar, S., Paswan, M. and Mahato, D. (2019) Chebyshev Operational Matrix Method for Lane-Emden Problem. Nonlinear Engineering, 8, 1-9. https://doi.org/10.1515/nleng-2017-0157

[25] El-Gamel, M. (2014) Numerical Comparison of Sinc-Collocation and ChebychevCollocation Methods for Determining the Eigenvalues of Sturm-Liouville Problems with Parameter-Dependent Boundary Conditions. SeMA Journal, 66, 29-42. https://doi.org/10.1007/s40324-014-0022-9

[26] Mason, J. and Handscom, D. (2003) Chebyshev Polynomials. CRC Press, Boca Raton. https://doi.org/10.1201/9781420036114 